\title{
Aortic dissection in a young male with isolated interrupted aortic arch: an unusual association
}

\author{
Shekhar Kunal ${ }^{1}$, Pooja Pathak $^{2}$, Shruti Mittal ${ }^{3}$, Vijay Pathak ${ }^{1}$, Prateek Vaswani ${ }^{4}$ \\ ${ }^{1}$ Department of Cardiology, Sawai Man Singh Medical College, Jaipur; ${ }^{2}$ Lady Health Officer, NIMS Medical College, \\ Jaipur; ${ }^{3}$ Department of Radiodiagnosis, Maulana Azad Medical College, New Delhi; ${ }^{4}$ Department of Cardiothoracic \\ and Vascular Surgery, All India Institute of Medical Sciences, New Delhi, India
}

\begin{abstract}
Interruption of the aortic arch (IAA) is a rare congenital anomaly which has been infrequently documented in the adult population. Aortic dissection (AD) is an emergency often presenting as acute chest pain and warranting urgent intervention. A 32-year-old male presented to Emergency Department with acute chest pain. Clinical examination revealed feeble pulses in bilateral lower limbs with a blood pressure difference between upper and lower limbs. Two-dimensional Echocardiography was suggestive of IAA with $\mathrm{AD}$ in the descending thoracic aorta. This was confirmed on computed tomography scan of the aorta which revealed complete interruption of the aortic arch distal to
\end{abstract}

Correspondence: Prof. Vijay Pathak, Department of Cardiology, Sawai Man Singh Medical College, Jaipur, Rajasthan, India.

E-mail: vijalp2000@gmail.com

Key words: Aortic dissection; D-dimer; echocardiography; interrupted aortic arch; multi detector Computed Tomography.

Authors' contributions: SK, VP, collected the clinical data; PP, review of the literature; SM, reviewed the radiological aspect; PV, reviewed the surgical aspects; SK, VP, PP, SM, PV, drafted the manuscript; SK, VP, worked on the concept and are responsible for the genuineness of the data as well as the guarantor of the paper. All the authors have read and approved the final manuscript.

Conflict of interest: The authors declare that they have no competing interests, and all authors confirm accuracy.

Ethics approval and consent to participate: No ethical committee approval was required for this case report by the Department, because this article does not contain any studies with human participants or animals. Informed consent was obtained from the patient included in this study.

Received for publication: 23 May 2020.

Accepted for publication: 17 November 2020.

${ }^{\circ}$ Copyright: the Author(s), 2021

Licensee PAGEPress, Italy

Monaldi Archives for Chest Disease 2021; 91:1404

doi: 10.4081/monaldi.2021.1404

This article is distributed under the terms of the Creative Commons Attribution Noncommercial License (by-nc 4.0) which permits any noncommercial use, distribution, and reproduction in any medium, provided the original author(s) and source are credited. left subclavian artery along with the presence of an intimo-medial flap in descending thoracic aorta. A diagnosis of IAA with AD was made. The patient underwent an urgent aortic repair surgery following which he has been largely asymptomatic. A suspicion of AD should always be made in any patient presenting with acute onset chest pain radiating to the back along with accelerated hypertension/feeble pulses in extremity. Occurrence of $\mathrm{AD}$ in an adult with IAA is a distinct rarity and has been documented only thrice.

\section{Introduction}

IAA refers to the complete lack of anatomical and luminal continuity between the ascending and descending aorta. This clinical entity, first described in 1778 by Raphael Steidele [1], accounts for about $1 \%$ of all congenital anomalies with an estimated annual incidence of 3 per million live births [2]. Celoria and Patton [3] in 1959 classified IAA into three distinct sub-types: Type A (43\%) where the interruption is distal to left sub-clavian artery, Type B (53\%) between left common carotid and left sub-clavian artery and Type C (4\%) between brachiocephalic and left common carotid artery. Most of the patients present during infancy with symptoms of congestive heart failure and has a high mortality rate in absence of a surgical intervention. IAA is infrequently encountered in the adult population as a majority of patients die during infancy [2]. Aortic dissection (AD) is often a medical emergency and refers to the disruption of medial layer of aorta due to a plane of cleavage leading to the formation of an intimo-medial flap and generation of true and false lumen [4]. The presence of AD in a patient with IAA complicates the disease course and alters its prognosis. IAA associated with $\mathrm{AD}$ has been previously reported thrice [5-7] in the medical literature. The paucity of the existing literature prompted us to report a 32year-old made with IAA and AD.

\section{Case Report}

A 32-year-old-male presented with complaints of retrosternal chest pain for past four hours which was sudden in onset, sharp, "tearing in nature" with radiation to the back and abdomen. He had never ever experienced such an episode of severe chest pain with no relief on using sub-lingual nitrate, which he had received prior to presentation. He also complained of profuse diaphoresis for the past 2 hours. The patient denied having fever, cough, visual or neurological impairment. There 
was no history of trauma/surgical intervention in recent past. He was recently diagnosed with hypertension and was on tablet Telmisartan $40 \mathrm{mg}$ for the past 3 months with poor compliance. He had been an ex-smoker (6 pack-years) and had quit 3 years back. On examination, his blood pressure readings were 180/94 $\mathrm{mm} \mathrm{Hg}$ and 186/90 $\mathrm{mm} \mathrm{Hg}$ in right and left upper arm respectively. However, his lower limb pulses were feeble and recorded a blood pressure of 110/66 mm Hg. Examination of cardiovascular system was unremarkable.

\section{Investigations}

Routine investigations including renal and liver function tests were within normal limits. A 12-lead-electrocardiograph was sug- gestive of left ventricular hypertrophy with strain pattern in leads V5 and V6. Serial cardiac troponin done on presentation and 6 hours later was negative. A quantitative D-dimer test was positive (1159 ng/mL ref: $<500 \mathrm{ng} / \mathrm{mL}$ ). Chest radiograph (postero-anterior view) revealed notching of inferior rib margin of the $2^{\text {nd }}$ and $3^{\text {rc }}$ bilateral ribs (Figure 1A). Two-dimensional Echocardiography (transthoracic view) reported normal biventricular function with concentric hypertrophy of left ventricle. In addition, supra-sternal view revealed interruption of aortic arch distal to left subclavian artery with absence of flow on Doppler imaging. Transesophageal Echocardiography (mid-esophageal descending aortic short axis view) reported presence of an intimo-medial flap in the descending aorta separating it into a true and false lumen (Figure 1B). There was no evidence of patent ductus arteriosus, ventricular septal defect or other intracardiac defects. An ECG-gated multi-detector computed tomography (MDCT) scan of aorta, revealed a complete

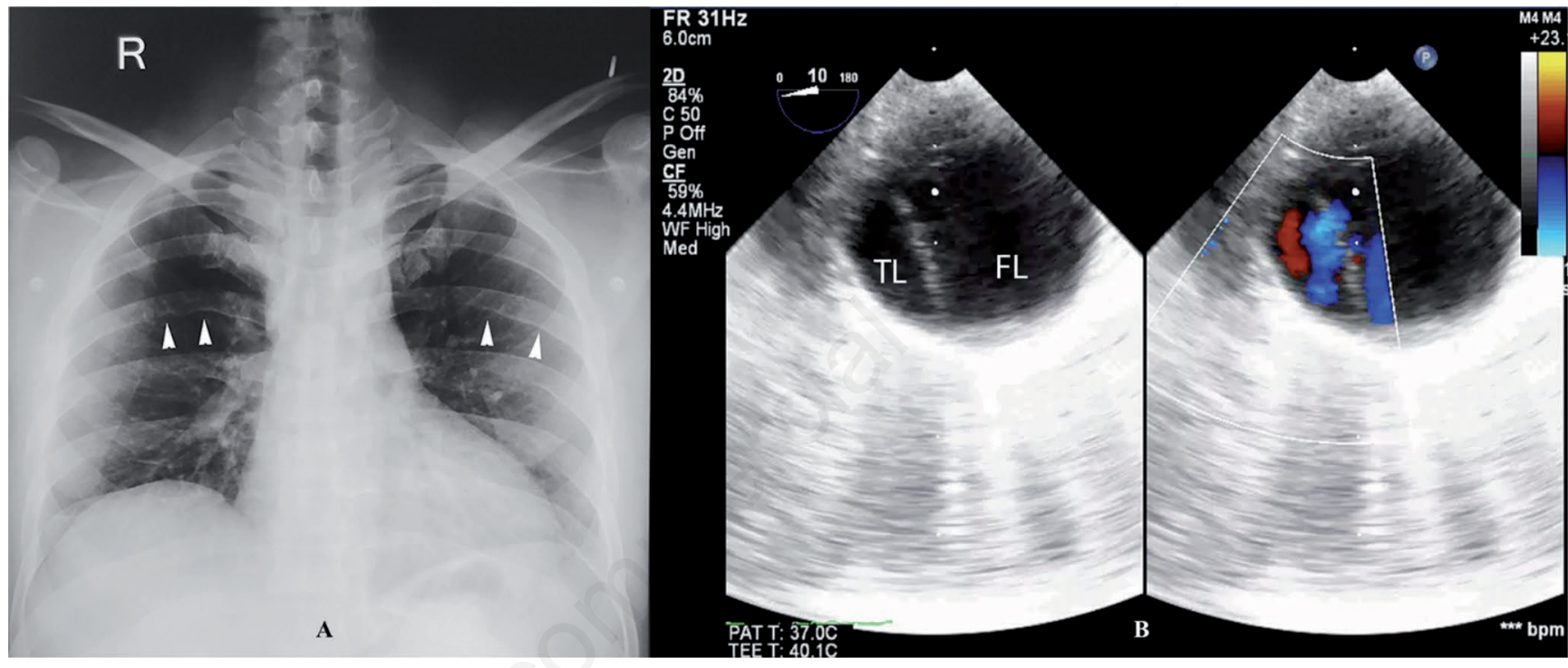

Figure 1. A) Chest radiograph postero-anterior view showing notching of the inferior rib margins (white arrowheads) of $2^{\text {nd }}$ and $3^{\text {rd }}$ rib bilaterally. B) Transesophageal echocardiography (mid-esophageal descending aortic short axis view) of the descending thoracic aorta showing the presence of an intimo-medial flap dividing the lumen into a smaller true lumen (TL) and a larger false lumen (FL). Colour Doppler images of the same revealed sluggish and decreased flow in the false lumen compared to that in the true lumen.

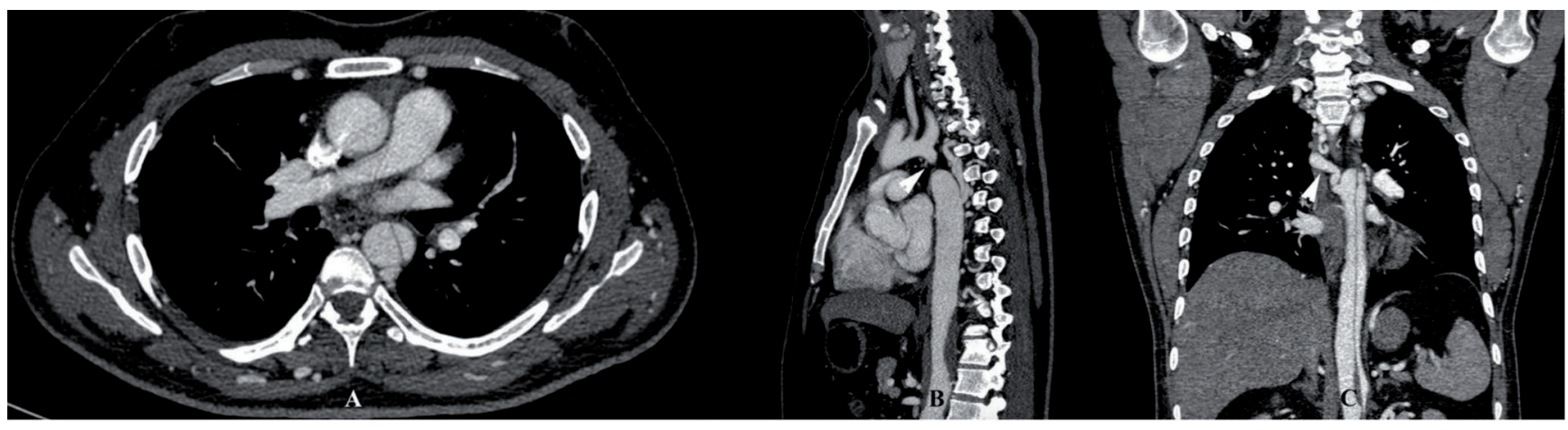

Figure 2. A) Computed tomography (CT) aortogram (axial view) showing the presence of an intimo-medial flap in the descending thoracic aorta dividing the lumen into a smaller true lumen and a larger false lumen. B) CT aortogram (sagittal view) showing a short segment complete interruption of the aortic arch (white arrowhead) just distal to the origin of left sub-clavian artery. C) CT aortogram (coronal view) showing the presence of a linear intimo-medial flap in the descending aorta extending distal to the interrupted segment. In addition, there is a presence of a large collateral channel (white arrowhead) supplying the interrupted segment of descending aorta. 
interruption ( $9 \mathrm{~mm}$ gap) in the aortic arch just distal to the origin of left subclavian artery (Figure 2). Multiple arterial collaterals were seen arising from bilateral sub-clavian artery, internal mammary artery along bilateral chest wall, axilla and upper para-vertebral region. In addition, there was a presence of an intimo-medial flap in the descending thoracic aorta (DTA) with the entry tear located at the ostia of a large collateral vessel $1.2 \mathrm{~cm}$ distal to the interrupted part of aorta. This dissection flap extended from the interrupted segment to aortic bifurcation (Figure 3). The origin of celiac axis, superior mesenteric, inferior mesenteric and left renal artery was from true lumen while right renal artery arose from false lumen. A diagnosis of isolated type A interrupted aortic arch with Stanford type B aortic dissection was made based on: (a) clinical presentation, (b) TTE and TEE echocardiographic views and confirmed on (c) CT aortogram along with multiplanar reconstruction. The patient was initially managed with beta-blockers along with ACE-inhibitors for control of heart rate and blood pressure. There was marked improvement in his symptoms over the next few days and a cardiothoracic surgical opinion was sought. Following stabilization with medical therapy, patient was referred to the Department of Cardiothoracic Surgery for further surgical repair. The patient underwent an aortic interposition graft repair using gelatin impregnated Dacron graft for the interrupted segment through a left anterolateral thoracotomy. In addition, large collaterals draining into DTA were clipped and divided. The dissection flap in DTA was partially excised with the distal end of

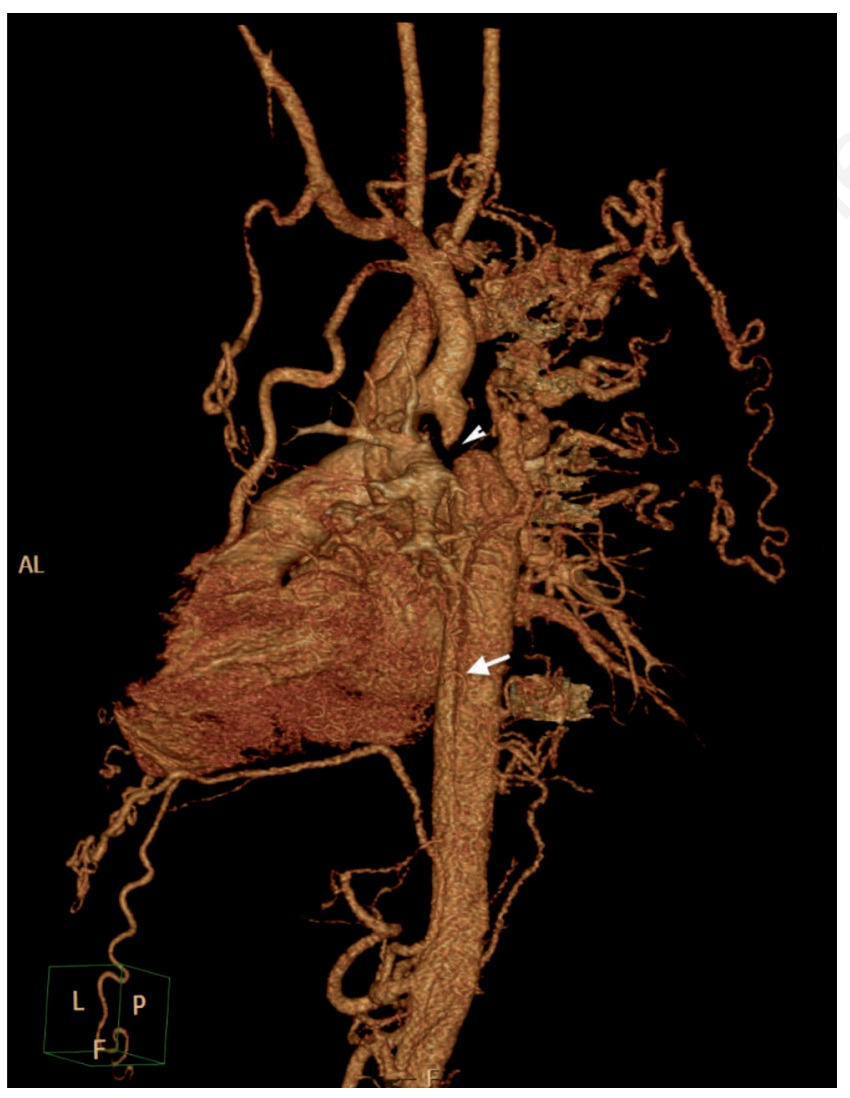

Figure 3. Three-dimensional reconstruction of the CT aortogram showing a short segment complete interruption of the aortic arch (white arrowhead) just distal to the origin of left sub-clavian artery along with the presence of a linear intimo-medial flap (white arrow) in the descending aorta distal to the interrupted segment. the graft segment anastomosed to DTA in a continuous manner such that both true and false lumen shall be perfused. He has been under follow-up for the past 1 year and has been markedly asymptomatic since then.

\section{Discussion}

IAA can be classified into three major forms based on associated defects: isolated, simple and complex. A majority of patients with IAA have an associated VSD (most common) and PDA who are then termed as simple IAA [8]. Complex IAA have associated complicated congenital anomalies such as double outlet right ventricle, truncus arteriosus and transposition of great arteries [8]. It is very unusual for a patient with an isolated IAA to survive infancy however, there have been a handful of reports documenting this adult form of IAA. A majority of adult subset of isolated IAA patients have type A IAA (79\%) with an extensive developed collateral circulation, a reason often cited for their survival [9]. The spectrum of clinical presentation in these patients range from symptoms of long standing hypertension to limb claudication, transient ischemic attacks and dizziness due to vertebral steal [2].

$\mathrm{AD}$ is often a medical emergency and patients usually present with complaints of acute onset chest pain as was seen in our patient too. The estimated annual incidence of $\mathrm{AD}$ is six per lakh with a higher rate seen in men and elderly [4]. The most common risk factor for $\mathrm{AD}$ is hypertension which is usually poorly controlled. In addition, pre-existing aortic diseases such as coarctation or aortic interruption often increases risk for AD. Two classification schema exists for AD viz. Debakey and Stanford classification with the latter being more popular and practical. Stanford type A refers to involvement of ascending aorta with or without affection of descending aorta while type B refers to isolated descending aortic involvement [4].

The diagnosis of both IAA and AD is initially based on a strong clinical suspicion followed by confirmatory findings on various imaging techniques. TTE/TEE forms the primary imaging modality and can easily demonstrate arch interruption/aortic intimal flap. However, false positive findings due to multiple artefacts limits its diagnostic potential. CT angiography along with multiplanar reconstruction is considered to be the gold standard for diagnosis of IAA as well as AD owing to its short scanning time, better spatial resolution and superior anatomical characterisation $[2,4]$

A search of the literature using the keywords "aortic dissection" and "interrupted aortic arch" in PubMed and IndMed database as well as Google search revealed three cases of IAA associated with $\mathrm{AD}$ previously reported in the medical literature. Li and Zheng [5] for the first time reported IAA with AD in a 37-year-old male who had presented with hypertension. CT angiography revealed a localised dissection of the aorta along with IAA. The authors had failed to classify the sub-type of IAA in this patient. Subsequently, Peng et al. [6] documented a 38-year-old male who presented with epigastric pain and was later diagnosed with isolated IAA and type B aortic dissection based on MDCT angiographic findings. Perez and colleagues [7] recently reported this association in a 55-year-old male with chest pain and dyspnoea on presentation. A diagnosis of type A IAA along with Stanford type A AD with BAV and hemopericardium was made. Our patient too presented with symptoms of chest pain along with a history of hypertension and was subsequently diagnosed with isolated type 
A IAA and type B AD making it the second case in the medical literature. We have also for the first time provided a detailed description of this association highlighting the utility of TTE and TEE along with MDCT angiography in the diagnosis of these patients.

The association of IAA with AD not only increases the risk of mortality but also magnifies chances of complications during aortic repair. The presence of multiple collateral channels at the proximal end of interrupted descending thoracic aorta increases the chance of AD. Peng et al. [6] had postulated that "blood stream from these collateral arteries could result in turbulent stream inside this interrupted aorta rather than laminar like that seen in normal aorta" which increases the chances of AD as could have been in our patient. The management of IAA is often surgical with extra-anatomic bypass or end to end/end to side anastomosis being the preferred technique. However, few reports do mention the role of percutaneous stent placement following perforation with a percutaneous wire [10]. Surgery in adults with IAA is often fraught with complications and procedural difficulties owing to a large number of collateral channels

AD should always be suspected in a patient presenting with chest pain, accelerated hypertension and/or decreased peripheral pulses. Though the outcome of adult form of IAA is good owing to the vast network of collaterals, surgery is often the treatment of choice. The co-existence of AD with IAA increases disease severity and warrants immediate surgical repair.

\section{References}

1. Steidele RJ. Samml Chir u Med Beob (Vienna) 1778;2:114.

2. Gordon EA, Person T, Kavarana M, Ikonomidis JS. Interrupted aortic arch in the adult. J Card Surg 2011;26:405-9.

3. Celoria GC, Patton RB: Congenital absence of the aortic arch. Am Heart J 1959;58:407-13.

4. Erbel R, Aboyans V, Boileau C, et al. ESC Committee for Practice Guidelines. 2014 ESC Guidelines on the diagnosis and treatment of aortic diseases: Document covering acute and chronic aortic diseases of the thoracic and abdominal aorta of the adult. The Task Force for the Diagnosis and Treatment of Aortic Diseases of the European Society of Cardiology (ESC). Eur Heart J 2014;35:2873-926.

5. Li J, Zheng M. Aortic arch interruption with thoracic aortic dissection. Ann Thorac Surg 2011;91:615.

6. Peng LQ, Yang ZG, Yu JQ, et al. Isolated interrupted aortic arch accompanied by type B aortic dissection and extensive collateral arteries diagnosed with MDCT angiography. Clin Imaging 2012;36:602-5.

7. Pérez TM, García SM, Velasco ML, Sánchez AP. Interrupted aortic arch diagnosis by computed tomography angiography and 3-D reconstruction: A case report. Radiol Case Rep 2017;13:35-8

8. Yang DH, Goo HW, Seo DM, et al. Multislice CT angiography of interrupted aortic arch. Pediatr Radiol 2008;38:89-100.

9. Sai Krishna C, Bhan A, Sharma S, et al. Interruption of aortic arch in adults: surgical experience with extra-anatomic bypass. Tex Heart Inst J 2005;32:147-50.

10. Goel PK, Moorthy N. Percutaneous reconstruction of interrupted aortic arch in an adult. JACC Cardiovasc Interv 2013;6: e21-2. 